\title{
Author's response to Harber et al. (2008)
}

\author{
David Allen Galbraith · David Weill
}

Published online: 30 July 2009

(C) Springer-Verlag 2009

\section{Erratum to: Int Arch Occup Environ Health DOI 10.1007/s00420-009-0431-8}

It is very unfortunate that the incorrect body text was typeset for "Author's response to Harber et al. (2008)". The correct text appears below.

Dear Editors of IAOEH (Hans Drexler, Editor-in-Chief, Karl Heinz Schaller, Associate Editor):

In response to Dr. Harber, Dr. Harrison and Dr. Gelb regarding their CDHS report (Harrison et al. 2006), we wish to clarify that our comments centered not on the association they reported of the two cases of lung disease with diacetyl or butter flavoring, but rather with the apparent certainty displayed by the authors regarding their assumed diagnoses of bronchiolitis obliterans. Instead of announcing in the title of the report the discovery of two additional flavorings workers with bronchiolitis obliterans, we believe it would have been more prudent to characterize these cases as being suspected of having this rare lung disease. We feel that they also should have devoted some attention to other disease processes that might also reasonably have been under consideration, that are known to present with similar clinical and radiographic findings.

The online version of the original article can be found under doi: 10.1007/s00420-009-0431-8.

D. A. Galbraith $(\square)$

Palo Alto Medical Foundation,

795 El Camino Real, Palo Alto, CA, USA

e-mail: dgalbraithmd@ hotmail.com

D. Weill

Stanford University Medical Center,

300 Pasteur Drive, Room H3143, Stanford, CA, USA
In Case 1, as we mentioned in our review, severe asthma, possibly related to occupational exposures, could have easily presented with an identical array of complaints, CT findings, and PFT results. While asthma is usually recognized to be responsive to bronchodilators, a substantial fraction of asthmatics are known to be refractory to bronchodilator therapy. Biopsy data would certainly have been helpful to provide more diagnostic certainty, particularly if more aggressive treatment was being considered for this individual.

In Case 2, we found it interesting that the unnamed expert pathologist would have interpreted the presence of eosinophilic infiltrates, together with noncaseating granulomas, as being "highly consistent" for bronchiolitis obliterans. The majority of lung pathologists, in our experience, would find this histologic story much more compelling to support a diagnosis of allergic alveolitis, a disease characterized by eosinophilic involvement and, indeed, the presence of interstitial granulomas and progressive fibrosis with continued exposure to the allergen in question. Constrictive bronchiolitis, on the other hand, is felt to result from an inflammatory and fibrogenic process of the membranous and respiratory bronchioles, eventually leading to progressive narrowing and obstruction of these distal airways. The lack of this kind of pathologic description and the failure of the authors to even mention a consideration of allergic alveolitis is an oversight, in our opinion.

We believe that the cause of severe lung disease in the population of flavorings workers has yet to be adequately explained. We simultaneously respect the efforts of NIOSH and other health authorities to use the information they have gained to better protect these workers and others possibly exposed to similar chemicals in the workplace. At the same time, we advise caution against rendering a certain diagnosis in the absence of sufficient, confirmatory clinical information. With the data provided in their 2006 report, the clear confidence Harber et al. displayed appears to us to be unwarranted. 